\title{
Residents call for duty hour reform
}

$\mathrm{O}$ utside of a reading of Snow White and the Seven Dwarves, the words "sleepy" and "doc" should not appear next to each other, Canadian medical residents contend.

The fatigue from working excessively long shifts is affecting the wellbeing and learning ability of medical residents, as well as putting patients at risk, and as a consequence, duty-hour reform is needed, according to the Canadian Association of Internes and Residents (CAIR).

"Providing patient care in a safe and ethical manner is intrinsic to professionalism," the association argues in a position paper, Canadian Patient and Physician Safety and Wellbeing: Resident Duty Hours, which urges adoption of a system that limits uninterrupted duty hours to 16 or less and provides for "adequate time in-between work periods to eliminate the effects of sleep deprivation." (www.cair.ca/u/elibrary /CAIR_EN_compressed\%20final\%20 Apr\%204.pdf).

"There seems to be mounting evidence related to the effects of resident fatigue, and we undertook to look over all the evidence to see how we could be doing this better," says Dr. Adam Kaufman, the association's president and a third-year resident in family and emergency medicine at the University of Toronto in Ontario.

In addition to recommending that resident duty hours be restricted in such a way that "they do not in any way endanger" patient or resident health, the position paper also recommends:

- Duty hours should be managed to optimize education.

- Residents should receive formal handover training.

- Duty hours "should be flexible enough to accommodate the specific context of the resident's role and the service needs on particular rotations."

- "The management of duty hours should parallel a change in the culture of medicine that addresses the effects and consequences of uninter-

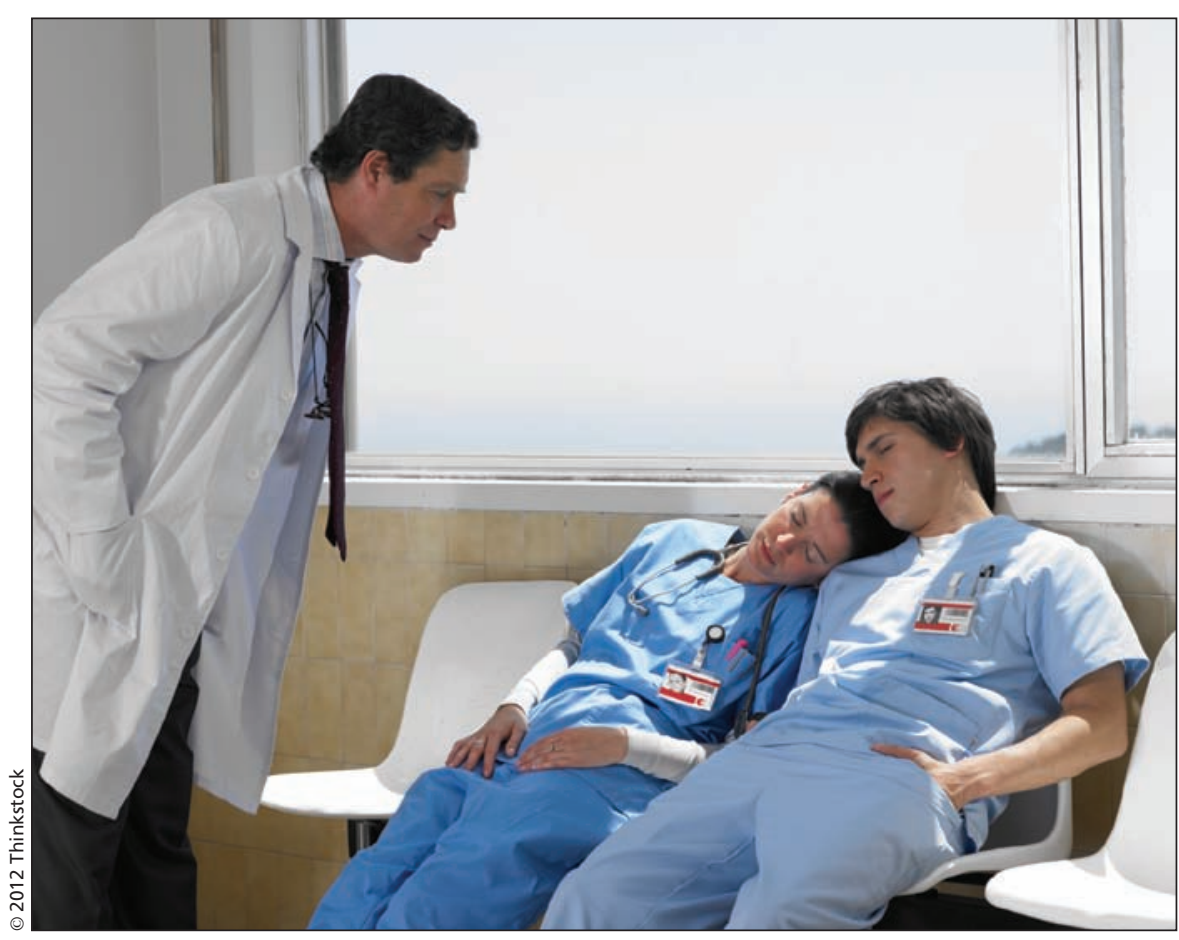

A 2011 survey of Canadian Association of Internes and Residents members indicated that $80 \%$ experienced "instances where the quality of their care had been compromised due to the number of consecutive hours they have been working."

rupted consecutive duty hours for the medical profession as a whole, including staff physicians and nonresident learners."

- "Where a violation of Federal or Provincial ethical, legislative, or legal standards has occurred, including but not limited to those related to the Canadian Charter of Rights and Freedoms, CAIR calls upon all stakeholders to address and remedy the situation as swiftly as possible."

Lengthy duty hours have come under assault in recent years as research revealed correlations between the incidence of medical errors and resident fatigue, as well as a higher incidence of vehicle crashes as exhausted residents commuted home (www.cmaj.ca/lookup /doi/10.1503/cmaj.090772). The United States Institute of Medicine proposed that a five-hour nap be made mandatory for all medical interns and residents who are putting in 16-hour shifts (www.cmaj.ca/lookup/doi/10.1503/cmaj
.109-3146). But defenders of lengthy duty hours for residents have long countered that limitations would compromise medical training by reducing clinical exposure at a time when it is most needed.

The international response to the concerns about patient and resident safety has varied, with Canada very much an outlier. A European Union working time directive compelled member nations to move toward a 48-hour week for residents, with a maximum of 13 consecutive duty hours. The US Accreditation Council for Graduate Medical Education approved new resident duty hour limits to restrict shifts to 16 hours for first-year residents and to 24 hours in subsequent years, with additional time for handovers.

Canada, by contrast, has not moved to limit resident duty hours, although the Royal College of Physicians and Surgeons of Canada is hoping to develop a pan-Canadian policy. 
The lack of alacrity with which change has occurred in Canada prompted medical residents in Quebec to launch a grievance in 2009 contending that the 24-hour on-call schedule permitted under their provincial contract constituted a violation of the Canadian Charter of Rights and Freedoms (www.cmaj.ca /lookup/doi/10.1503/cmaj.090527). An arbitrator subsequently ruled that 24hour resident shifts in Quebec hospitals had to be reduced to maximum of 16 hours but that ruling is being appealed.

Kaufman says CAIR isn't wedded to any specific hourly limit for residents, except one that substantially reduces the length of consecutive duty hours. Research has shown that serious negative effects on residents and patients occur when shifts reach 24 hours in length, he says, adding that while there is no "magic number at which everything is solved," the research also suggests that 16 hours is a reasonable maximum.

"We want to spark a dialogue at the national level and see if that trickles down. It's about making use of the resources we've got in the most effective way possible. What we really need are on-the-ground, local solutions - optimal resource usage with a local solution."

There's no question a national debate and a national consensus is needed, says Dr. Kevin Imrie, vicepresident of education for the Royal College of Physicians and Surgeons of Canada. "There is no one agreed-upon configuration, and that has to be part of the discussion," he notes, adding that it is important there be respect in the profession of medicine for physician wellness and that no one is "belittled for respecting duty-hour rules or having a life outside of medicine."

For their part, national and provincial associations indicate they are amenable to discussion about CAIR's proposals.

"We feel that it is a well thought-out paper that takes into consideration both the residents' needs and the pedagogical concerns of their faculty," Dr. Sarkis Meterissian, chair of the Association of Faculties of Medicine of Canada's Standing Committee on Postgraduate Medical Education, writes in an email. "They have accurately identified the areas that require special attention, namely handovers and educational requirements. We look forward to working with CAIR both nationally and regionally to arrive at a workable approach to resident work-hours."

Similarly, the Ontario Hospital Association wrote in an email that "we know that similar practices are being explored in other jurisdictions and would want to ensure patient safety and quality of care remain top focal points."

But resolving the issue will be tricky and reducing the working hours of residents may result in new problems, says Dr. Najib Ayas, a researcher and clinician with Vancouver Coastal Health's Sleep Disorders Program in British Columbia. "There is always a balance. Having people stay up longer than 24 hours and caring for sick patients is probably not a good idea, but if you are having people switch often, there will be a downside to that as well."

In addition to the potential for increased errors due to discontinuity, other possible negative impacts include reduced clinical exposure and inadequate preparation for independent practice, Ayas wrote in a recent paper exploring "the good, the bad and the ugly" of restricting resident work hours (Crit Care Med 2012;40:960-6).

Many hospitals rely heavily on residents, especially at night, and gaps in coverage may emerge if they work fewer hours, Ayas says. "The reality is that, at any given hour, there will be fewer residents available to patients." — Roger Collier, CMAJ

CMAJ 2012. DOI:10.1503/cmaj.109-4187 